\title{
Eficacia del cruce de manos para producir analgesia en pacientes con dolor crónico de la extremidad superior
}

\author{
I. Jorquera Cáceres, D. Ilaja Cid, C. Magaña Aguilar, M. Tabilo Ponce, S. Jiménez Torres y H. Núñez Montenegro
}

Escuela de Kinesiología. Facultad de Salud. Universidad Santo Tomás. Arica, Chile

\section{ABSTRACT}

Background: Recent studies report that patients with chronic pain, they undergo changes in the processing of information that reaches them from the in which the stimuli are exercised, for example, when their hands are crossed. In patients with chronic pain, the presence of alterations in their somatotropic frames of reference has been demonstrated, and this is why the strategy of crossing the extremities through the midline could generate changes in the perception of pain.

Objective: To evaluate if an analgesic physiotherapy program with hand crossing technique is more effective than a program without crossing hands, in order to reduce pain.

Material and method: Sixteen patients with chronic pain in elbow, wrist or hand, were randomized in two groups. One received transcutaneous electrical nerve stimulation (TENS) with hand crossing and closed eyes, and the other (control group) received only the application of TENS. Each group was evaluated before and after the intervention with the McGill pain questionnaire and the visual analog scale (VAS).

Results: A decrease in post-intervention pain was observed in both groups; where the control group decreased an average of $3.5 \mathrm{~cm}$ and the experimental group $3.75 \mathrm{~cm}$. All sessions of the experimental group were statistically significant $(P=<0.05)$, while one session in the control group was not statistically significant $(P=0.051)$. However, the Analysis of variance (ANOVA) between both groups, did not give statistically significant ( $p=0.863)$.

Conclusions: The results do not show that the crossing of hands potentiates the effects of analgesic therapy on pain intensity. More information is the effects to include this technique as a support strategy periphery with the mere fact of changing the order

\section{RESUMEN}

Antecedentes: Estudios recientes refieren que los pacientes con dolor crónico sufren cambios en el procesamiento de la información que les llega desde la periferia con el solo hecho de cambiar el orden en que los estímulos les son ejercidos, por ejemplo, cuando se les cruzan las manos. En los pacientes con dolor crónico se ha demostrado la presencia de alteraciones en sus marcos de referencia somatotópicos, y es por esto que la estrategia de cruzar las extremidades por la línea media, podría generar cambios en la percepción dolorosa.

Objetivo: Evaluar si un programa de fisioterapia analgésica aplicada con cruce de manos es más eficaz que uno sin el cruce para disminuir el dolor.

Material y método: 16 pacientes con dolor crónico de codo, muñeca o mano ingresaron al estudio. Fueron aleatorizados en un grupo experimental, que recibió la aplicación de electroanalgesia nerviosa transcutánea (TENS) con cruce de manos y ojos cerrados, además de un grupo control, que recibió solo electroanalgesia. A cada grupo se les evaluó su dolor pre y postintervención, con el cuestionario del dolor de McGill y la escala visual análoga [EVA].

Resultados: Ambos grupos, el control y experimental, presentaron una disminución en el dolor postintervención; el grupo control disminuyó una media de $3,5 \mathrm{~cm}$ y el grupo experimental con una media de $3,75 \mathrm{~cm}$. Todas las sesiones del grupo experimental fueron estadísticamente significativas $(p<0,05)$, mientras que el grupo control presentó una sesión no estadísticamente significativa $(p=0,051)$. El análisis de varianza (ANOVA) entre ambos grupos, sin embargo, no dio estadísticamente significativo ( $p=0,863)$.

Conclusiones: Los resultados no evidencian convincentemente que el cruce de manos potencia los efectos de la terapia analgésica en relación con la intensidad dolorosa. Más estudios serían necesarios para incluir esta técnica como una estrategia de apoyo en pacientes con dolor cró-

Recibido: 23-03-2018

Aceptado: 20-01-2019

Jorquera Cáceres I, Ilaja Cid D, Magaña Aguilar C, Tabilo Ponce M, Jiménez Torres $S$ y Núñez Montenegro H. Eficacia del cruce de manos para producir analgesia en pacientes con dolor crónico de la extremidad superior. Rev Soc Esp Dolor 2019;26(2):81-88.
Correspondencia: Ivonne Jorquera Cáceres ivonnejorquera@santotomas.cl 
in patients with chronic pain, and to manage sensory alterations and changes in the processing of information that reaches the periphery.

Key words: Chronic pain, analgesic physiotherapy, hand crossing, upper extremity injury. nico, y poder manejar así las alteraciones sensoperceptivas y los cambios en el procesamiento de la información que les llega de la periferia.

Palabras clave: Dolor crónico, fisioterapia analgésica, cruce de manos, lesión de extremidad superior.

\section{INTRODUCCIÓN}

La asociación internacional para estudio del dolor (IASP) define dolor como una experiencia emocional o sensitiva desagradable y que no necesita estar presente una lesión tisular física en el individuo. Esta misma asociación manifiesta que el dolor crónico es aquel que dura más de tres a seis meses, incluyendo habiéndose realizado los tratamientos adecuados (1).

El dolor crónico es un trastorno que persiste o se repite en el tiempo, y representa un cuadro multifactorial. Estudios recientes refieren que los pacientes que lo sufren presentan una reorganización funcional de su corteza somatosensorial y motora primaria, y que la representación de sus segmentos del cuerpo cambiaría a mayor cronicidad (2-9). Pacientes con lesiones crónicas de extremidad superior, específicamente síndrome doloroso regional complejo, presentarían disminuida su capacidad de determinar el orden en que los estímulos nociceptivos o táctiles les son ejercidos cuando estos son realizados con el cruce de las manos más allá de la línea media. Además, han referido menos dolor y mayor temperatura con esta misma estrategia, lo que se revierte al volver a colocar sus extremidades a los lados del cuerpo. Esto se ha interpretado como que en los pacientes con dolor crónico sus marcos de referencia somatotópicos a nivel cortical han sufrido cambios en el procesamiento desde la información que les llega de sus tejidos, y que este cambio en las percepciones dolorosas podrían ser utilizadas a favor de tratamientos rehabilitadores como la terapia física (3-5). El objetivo de este estudio fue evaluar si un programa de fisioterapia analgésica aplicada con cruce de manos es más eficaz que su aplicación sin el cruce de estas, en pacientes con dolor crónico de codo, mano o muñeca.

\section{PACIENTES Y MÉTODOS}

\section{Diseño del estudio y normas éticas}

Se realizó un ensayo clínico aleatorizado. Participaron 16 pacientes que ingresaron a la Clínica Kinésica de la Universidad Santo Tomás, sede Arica, durante los meses de septiembre y octubre del 2017, con dolor crónico en codo, muñeca o mano. A todos los participantes se les explicó los alcances del estudio y se les pidió firmar una carta de consentimiento previo a su ingreso al estudio. Esta investigación se desarrolló de acuerdo a los requerimientos de la declaración de Helsinki y las normas bioéticas del comité de ética de la Universidad Santo Tomás Macro Zona Norte.

\section{Criterio de inclusión y exclusión}

Criterios de inclusión: pacientes con edad entre 18 y 70 años, con patología musculoesquelética de extremidad superior de codo, muñeca o mano, mayor o igual a seis meses y con un dolor de tipo nociceptivo [causa mecánica como etiología del dolor), que presenten una intensidad del dolor en el momento de ingreso al estudio de una puntuación mayor o igual a 3/10, según la escala visual análoga (EVA].

Criterios de exclusión: pacientes con patología aguda (menor a tres meses de sintomatología), o dolor de origen neurológico (evidenciado con examen de velocidad de conducción]. Presencia de enfermedad cardiaca o arritmias, epilepsia, heridas abiertas o cirugías en extremidad superior de evolución menor a tres meses, pacientes embarazadas, con algún trastorno psiquiátrico diagnosticado o alergia a elementos fisioterapéuticos [gel conductor].

\section{Variables de valoración}

Los participantes fueron aleatorizados en dos grupos: grupo A (grupo control) y grupo $B$ (grupo experimental). Para esto se usó un sistema de sobres oscuros que contenían dentro designada la letra de cada grupo. El investigador encargado del ingreso de los pacientes y el analista estadístico fueron ciegos a la asignación de cada grupo.

Los participantes entraban a una sala ambientada especialmente para la sesión de tratamiento y se sentaban cómodamente. Antes de comenzar la intervención se les realizó una evaluación de sensibilidad superficial y se les limpió la zona de colocación de los electrodos para prevenir lesiones. El programa de analgesia aplicada con TENS (electroanalgesia nerviosa transcutánea), se desarrolló con el equipo portátil Modelo Care Tec IV, y correspondió a 5 sesiones de 20 minutos cada uno, modalidad tipo acupuntura (baja frecuencia), onda cuadrática bipolar, con duración de pulso de 250 us y frecuencia de $5 \mathrm{~Hz}$, a una intensidad de contracciones visibles y sensación de parestesia eléctrica. Los electrodos se colocaron sobre un miotoma del área dolorosa 
de extremidad superior de cada paciente (Figura 1). Antes del comienzo del experimento, los participantes se familiarizaron con la estimulación sensorial de la corriente eléctrica (5 minutos). Durante el experimento, se les pidió a los participantes del grupo control que mantuvieran las extremidades superiores sin cruzar, y al grupo experimental cruzarlas sobre la línea media del cuerpo, además de utilizar un antifaz sobre sus ojos con el fin de bloquear su campo visual (Figura 1). La distancia entre las manos era la misma, alrededor de $40 \mathrm{~cm}$ en las 2 condiciones, cruzadas o no cruzadas.

Previo a iniciar su primera sesión, y al finalizar el programa de 5, a cada sujeto se le midió su intensidad del dolor con la escala visual análoga (EVA) (10) y valoró sus características, con el cuestionario de dolor de McGill (11). Para la intensidad, los participantes fueron explícitamente instruidos para colocar una línea en la escala, que iba desde calificación 0 en respuesta a un "sin dolor" y 10 (final de la escala) a "la sensación dolorosa más fuerte que se pueda imaginar". Antes y después de cada sesión también se les preguntó la intensidad percibida de su dolor con la escala EVA.

Para la valoración de las características del dolor que presentaban los pacientes pre y postexperimento, se les pidió a los participantes elegir dentro de los 78 adjetivos del cuestionario de Mcgill, los que más se acercaran a su experiencia dolorosa en el último día.

\section{Análisis estadístico}

Para verificar si las diferencias entre los resultados pre y postintervención entre sesiones del grupo control y el grupo experimental eran estadísticamente significativas, se aplicó un análisis estadístico con T de Student al 0,05\%. Para valorar las diferencias entre ambos grupos y su posición de sus extremidades (cruzado y no cruzado), se aplicó un análisis de varianza (ANOVA) al $0,05 \%$ con el programa estadístico Mystat 12 versión 12.02.00 del año 2007.

\section{RESULTADOS}

Se incluyeron 16 pacientes en el estudio: 8 (1 hombre y 7 mujeres) constituyeron el grupo control (edad media de $42 \pm 14,3$ ); y 8 (3 hombres y 5 mujeres] conformaron el grupo experimental (edad media de 36 $\pm 14,8$ ). Detalles de las intensidades de dolor percibidas por los participantes pre y postintervención son expuestas en la Tabla I.

En ambos grupos las intensidades del dolor disminuyeron postintervención en cada sesión. El grupo experimental inició con una media de $4,87 \mathrm{~cm}$ en la primera sesión y finalizó la quinta, con una media de un $1,12 \mathrm{~cm}$ (el paciente con menos dolor ingresó con un EVA de 4, y el paciente con más dolor con un EVA de 8). El grupo control inició con una intensidad dolorosa de $4,75 \mathrm{~cm}$ y finalizó con una media de 1,25 cm (el paciente con menos dolor ingresó con un EVA de 3 y el paciente con más dolor con un EVA de 7]. El estadístico de Levene fue utilizado para analizar la homogeneidad entre los grupos, evidenciándose con un resultado de 0,384 (Tabla II).

En todas sus sesiones el grupo experimental, presentó diferencias estadísticamente significativas ( $p<0,05$ ) (Tabla I y Figura 2). El grupo control, en una de sus sesiones la diferencia entre pre y post, no fue estadísticamente significativa ( $p=0,051$ ) (Tabla I y Figura 3). El análisis de varianza (ANOVA) entre ambos grupos no dio estadísticamente significativo ni para la sesión 1 ( $p=0,863)$, ni para la sesión 5 ( $p=0,868$ ) (Tabla II).

En relación con las dimensiones de dolor (Tablas III y IV), según el cuestionario del dolor de McGill (1975), el grupo experimental presentó un cambio en cuanto al índice de valoración del dolor (PRI). Preintervención, los pacientes manifestaron al menos una característica de la dimensión sensorial (62,5\%) y dimensión miscelánea (25\%), y postintervención disminuyeron la dimensión miscelánea (12,5\%) y aumentaron su dimensión sensorial (75\%). En el grupo control, al momento de la preintervención, un alto porcentaje de los pacientes describió características para la dimen-

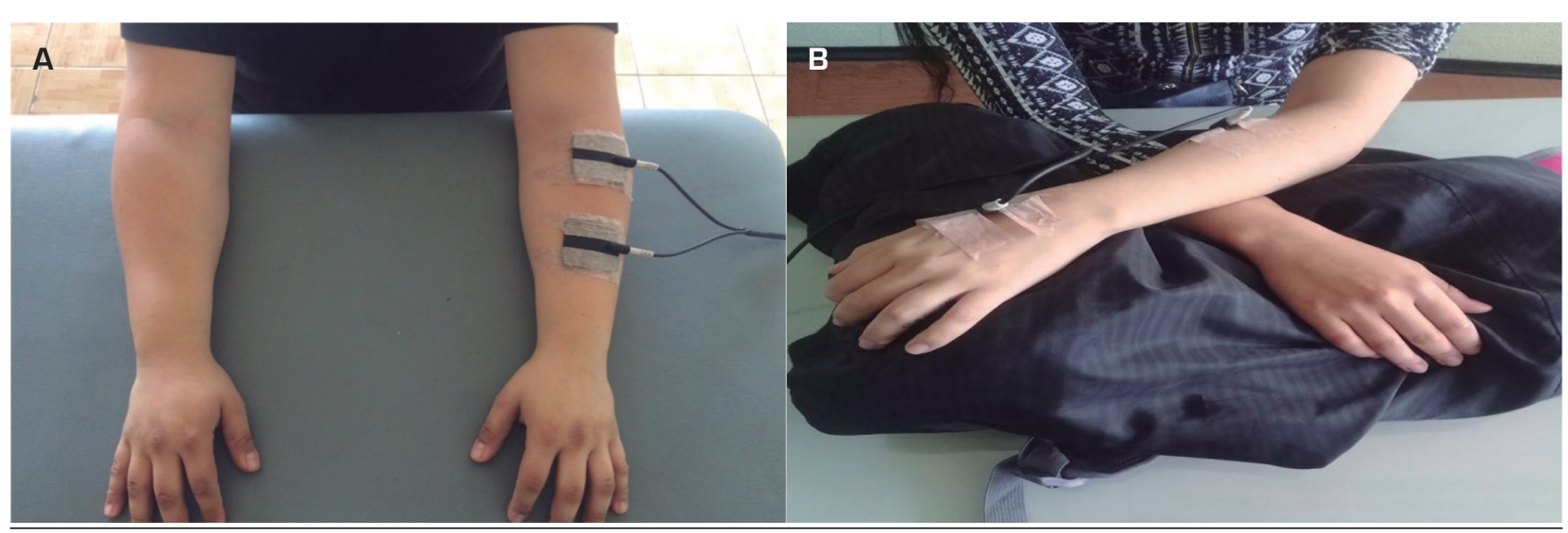

Fig. 1. Lado izquierdo: grupo A (grupo control sin cruce de manos). Lado derecho: grupo B (grupo experimental con cruce de manos]. 
TABLA I

DOLOR GRUPO CONTROL Y EXPERIMENTAL PRE Y POSTSESIÓN, SEGÚN LA ESCALA VISUAL ANÁLOGA (EVA) Y MODELO ESTADÍSTICO

\begin{tabular}{|c|c|c|c|}
\hline \multicolumn{4}{|c|}{ Grupo control } \\
\hline Sesión & Media presesión (cm) & Media postsesión [cm] & Diferencia \\
\hline \multirow[t]{2}{*}{ Sesión 1} & 4,75 & 3,625 & 1,125 \\
\hline & $\mathrm{T}: 7$ & P: 0,051 & IC: $95,00 \% 0,009$ to 2,259 \\
\hline \multirow[t]{2}{*}{ Sesión 2} & 3,625 & 2,375 & 1,25 \\
\hline & $\mathrm{T}: 7,638$ & P: 0,000 & IC: $95,00 \% 0,863$ to 1,637 \\
\hline \multirow[t]{2}{*}{ Sesión 3} & 3,75 & 2,375 & 1,375 \\
\hline & $\mathrm{T}: 4,245$ & P: 0,004 & IC: $95,00 \% 0,609$ to 2,141 \\
\hline \multirow[t]{2}{*}{ Sesión 4} & 2,875 & 2,125 & 0,75 \\
\hline & $\mathrm{T}: 3,000$ & P: 0,020 & IC: $95,00 \% 0,159$ to 1,341 \\
\hline \multirow[t]{2}{*}{ Sesión 5} & 2,75 & 1,25 & 1,5 \\
\hline & $\mathrm{T}: 4,583$ & P: 0,003 & IC: $95,00 \% 0,726$ to 2.274 \\
\hline \multicolumn{4}{|c|}{ Grupo experimental } \\
\hline Sesión & Media presesión $(\mathrm{cm})$ & Media postsesión $[\mathrm{cm}]$ & Diferencia \\
\hline \multirow[t]{2}{*}{ Sesión 1} & 4,875 & 3 & 1,875 \\
\hline & $\mathrm{T}: 3,071$ & P: 0,018 & IC: $95,00 \% 0,431$ to 3,319 \\
\hline \multirow[t]{2}{*}{ Sesión 2} & 4,375 & 2,25 & 2,125 \\
\hline & T: 2,862 & P: 0,024 & IC: $95,00 \% 0,369$ to 3,881 \\
\hline \multirow[t]{2}{*}{ Sesión 3} & 3 & 1,87 & 1,13 \\
\hline & $\mathrm{T}: 3,211$ & P: 0,015 & IC: $95,00 \% 0,296$ to 1,954 \\
\hline \multirow[t]{2}{*}{ Sesión 4} & 2,87 & 1,75 & 1,12 \\
\hline & T: 3,813 & P: 0,007 & IC: $95,00 \% 0,427$ to 1,823 \\
\hline \multirow[t]{2}{*}{ Sesión 5} & 2,5 & 1,125 & 1,375 \\
\hline & $\mathrm{T}: 2,986$ & P: 0,020 & IC: $95,00 \% 0,286$ to 2,464 \\
\hline
\end{tabular}

TABLA II

FACTOR ANOVA. DIFERENCIAS ENTRE LOS GRUPOS EXPERIMENTAL Y CONTROL

\begin{tabular}{|c|c|c|c|c|c|c|}
\hline & & $\begin{array}{c}\text { Suma de } \\
\text { cuadrados }\end{array}$ & gl & $\begin{array}{c}\text { Media } \\
\text { cuadrática }\end{array}$ & $F$ & Sig. \\
\hline EVAIS 1 & Entre grupos & 0,063 & 1 & 0,063 & 0,031 & 0,863 \\
\hline EVAFS5 & Entre grupos & 0,063 & 1 & 0,063 & 0,029 & 0,868 \\
\hline \multicolumn{7}{|c|}{ Prueba de homogeneidad de varianzas } \\
\hline EVAlS 1 & Estadístico de Levene & gl1 & gl2 & Sig. \\
\hline EVAFS5 & 0,808 & 1 & 14 & 0,384 \\
\hline
\end{tabular}

Estadístico Levene muestra homogeneidad entre los grupos. Las diferencias entre los grupos para la sesión 1 y sesión 5 no fueron estadísticamente significativas ( $p>0,05)$. 
Grupo experimental

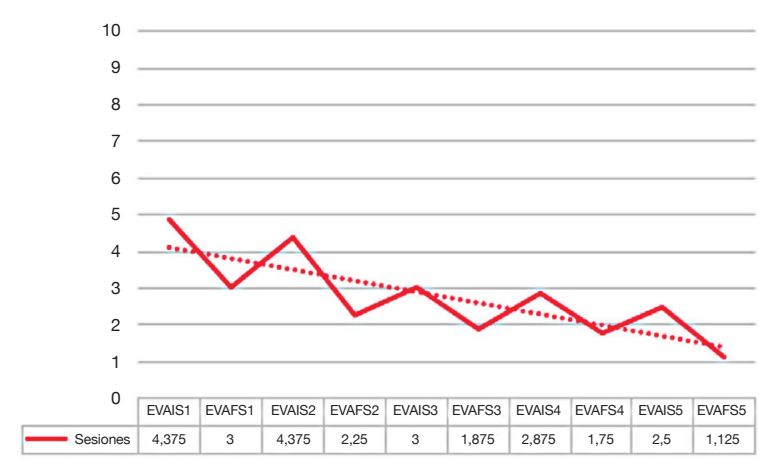

Este gráfico de línea muestra el análisis de la media calculada del grupo experimental (de ocho pacientes). En el eje $X$ se encuentran las intervenciones realizadas y la media obtenida durante las cinco sesiones, mientras que en el eje $Y$ se encuentra la escala visual análoga. Se puede observar que el EVA inicial de la sesión I (EVAIS1) y el EVA final de la sesión I (EVAFS1) durante las cinco sesiones realizadas en la clínica Santo Tomás Arica, que la línea tuvo una tendencia al descenso, con un inicio de una media de EVAIS1 de 4,875 en la primera sesión y en la quinta sesión con una media de un EVAFS5 de 1,125.

Fig. 2. Dolor grupo experimental pre y postsesión, según la escala visual análoga (EVA).

TABLA III

DISTRIBUCIÓN EN PORCENTAJES PARA EL ÍNDICE DE VALORACIÓN DEL DOLOR PREINTERVENCIÓN INICIAL Y POSTINTERVENCIÓN FINAL, SEGÚN EL CUESTIONARIO DEL DOLOR (PRI) DE MCGILL PARA EL GRUPO CONTROL

\begin{tabular}{|c|c|c|}
\hline PRI & Porcentaje pre & $\begin{array}{c}\text { Porcentaje } \\
\text { post }\end{array}$ \\
\hline $\begin{array}{c}\text { Sensorial } \\
\text { (1-10) }\end{array}$ & $50 \%$ & $0 \%$ \\
\hline $\begin{array}{c}\text { Afectivo } \\
\text { (11-15) }\end{array}$ & $25 \%$ & $37,50 \%$ \\
\hline Evaluativo (16) & $0 \%$ & $12,50 \%$ \\
\hline $\begin{array}{c}\text { Miscelánea } \\
\text { (17-20) }\end{array}$ & $25 \%$ & $50 \%$ \\
\hline
\end{tabular}

sión sensorial (50\%) y solo una pequeña parte al menos una característica para la dimensión afectiva (25\%) y dimensión miscelánea (25\%). Al finalizar el tratamiento, ningún paciente manifestó características de la dimensión sensorial [0\%), pero sí refirieron en la dimensión miscelánea (50\%), afectiva [37,5\%) e, incluso, evaluativa (12,5\%).

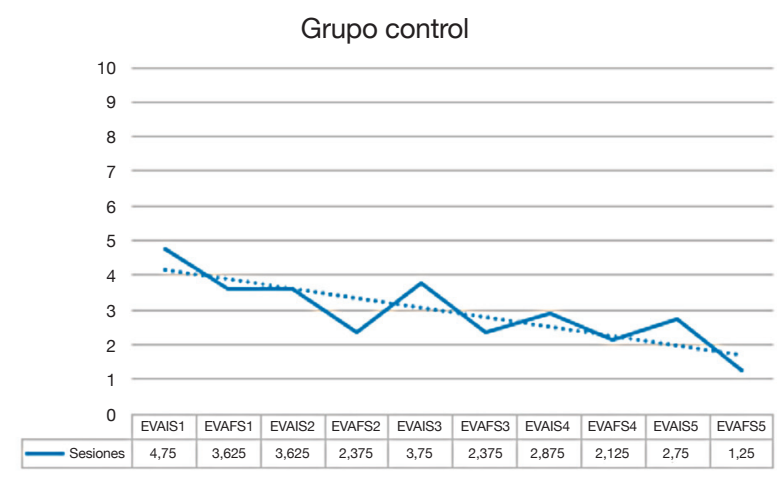

Este gráfico de línea muestra el análisis de la media calculada del grupo control (de ocho pacientes). En el eje $X$ se encuentran las intervenciones realizadas y la media obtenida durante las cinco sesiones. Mientras que en el eje $Y$ se encuentra la escala visual análoga. Se puede observar que el EVA inicial de la sesión I (EVAIS1) y el EVA final de la sesión I (EVAFS1) durante las cinco sesiones realizadas en la clínica Santo Tomás Arica que la línea tuvo una tendencia al descenso, con un inicio de una media de EVAIS1 de 4,75 en la primera sesión y en la quinta sesión con una media de un EVAFS5 de 1,25.

Fig. 3. Dolor grupo control pre y postsesión según la escala visual análoga (EVA).

TABLA IV

DISTRIBUCIÓN EN PORCENTAJES PARA EL ÍNDICE DE VALORACIÓN DEL DOLOR PREINTERVENCIÓN INICIAL Y POSTINTERVENCIÓN FINAL EN EL GRUPO EXPERIMENTAL SEGÚN EL CUESTIONARIO DEL DOLOR (PRI) DE MCGILL

\begin{tabular}{|c|c|c|}
\hline PRI & Porcentaje pre & $\begin{array}{c}\text { Porcentaje } \\
\text { post }\end{array}$ \\
\hline $\begin{array}{c}\text { Sensorial } \\
\text { (1-10) }\end{array}$ & $62,50 \%$ & $75 \%$ \\
\hline $\begin{array}{c}\text { Afectivo } \\
\text { (11-15) }\end{array}$ & $0 \%$ & $12,50 \%$ \\
\hline Evaluativo (16) & $12,50 \%$ & $0 \%$ \\
\hline $\begin{array}{c}\text { Miscelánea } \\
\text { (17-20) }\end{array}$ & $25 \%$ & $12,50 \%$ \\
\hline
\end{tabular}

\section{DISCUSIÓN}

En este estudio se hipotetizó que el cruzar las manos por la línea media dificulta el procesamiento somatosensorial en los pacientes con dolor crónico, y que esto podría ser aprovechado para tener un mayor efecto en la aplicación de una terapia física, y así disminuir el dolor. 
Gallace y cols. (4), en su estudio "Efectos Analgésicos con el cruce de brazos" desarrollado el año 2011 , demostró que, al cruzar los brazos sobre la línea media del cuerpo, los pacientes presentaron disminuida su intensidad de sensaciones táctiles y dolorosas percibidas cuando les entregaron estímulos eléctricos no nociceptivos y láser nociceptivo en sus manos. Sus resultados se justificaron fisiológicamente evaluando la actividad cerebral evocada de los pacientes, encontrando que para poder concienzar la localización de estímulos táctiles, no solo es primordial el rol de la corteza somatosensorial específica, sino también de áreas cerebrales que responden a estímulos pertenecientes a diferentes modalidades sensoriales [áreas como la límbica o las de asociación), y que, al cruzar los brazos se interfiere principalmente este procesamiento multimodal somatosensorial (4). Sambo y cols., el año 2013, siguiendo con esta línea de investigación, encontraron no solo déficits en la localización e intensidad, sino también en la capacidad de juzgar correctamente el orden de los estímulos táctiles ejercidos al cruzar las extremidades, denominándolo como "déficit de manos cruzadas" (3).

La habilidad para localizar donde un estímulo nociceptivo se está produciendo es fundamental para interactuar con el mundo y para la sobrevivencia. Sin embargo, en pacientes con dolor crónico se ha evidenciado que esta respuesta se encuentra alterada, incluso magnificada, lo que explicaría muchas veces las manifestaciones observadas en afecciones como el síndrome doloroso regional complejo, dolor fantasma, o síndrome de dolor lumbar crónico $(2-6,9)$. Nosotros quisimos probar que el cruzar las manos podría ser aprovechado en el caso de un paciente con dolor crónico, ya que al generar este "déficit de manos cruzadas", se podría intervenir con un medio físico la extremidad afectada y potenciar sus efectos moduladores del dolor. Nuestros resultados no pudieron confirmar lo planteado, aunque la intensidad del dolor disminuyó significativamente en todas las sesiones aplicadas al grupo experimental y no así en el grupo control. Al analizar las diferencias entre grupos (con análisis de varianza ANOVA) esta diferencia no fue estadísticamente significativa a favor del cruce de manos.

En la Figura 4, los valores de dolor del mayor porcentaje de pacientes del grupo control se encontraban entre 2,4 y $3,6 \mathrm{~cm}$, lo cual nos indica que estos pacientes se mantuvieron en un rango moderado de dolor, mientras que los del grupo experimental (Figura 5), al final de las 5 sesiones se encontraban entre los 2 y $3 \mathrm{~cm}$, evidenciando que estos pacientes disminuyeron a un rango de dolor leve al finalizar la intervención, y considerando que el grupo experimental comenzó incluso con una media de intensidad de dolor mayor. Este resultado es muy alentador para nuestra hipótesis. Estos resultados son similares a los presentados por Gallace y cols. (2011) (4), que nos indica que la técnica de cruce de manos es efectiva para influir en la percepción del dolor a nivel central. También estos resultados se justificarían con la idea de Agliotti (1999) (12), donde señala que cruzar las extremidades por la línea media del cuerpo reduciría la capacidad de determinar qué mano fue estimulada primero, por lo tanto, las posibilidades de reconocer el lado derecho o izquierdo serían nulas tomando esta idea como una ventaja para poder confundir el cerebro.

En relación con las dimensiones de dolor, aunque esto no era una variable de interés primaria en el estudio, se observaron importantes cambios: el grupo experimental manifestó postintervención mayores características en la dimensión sensorial, evidenciando una disminución en cuanto al tipo de dolor, y el gru-

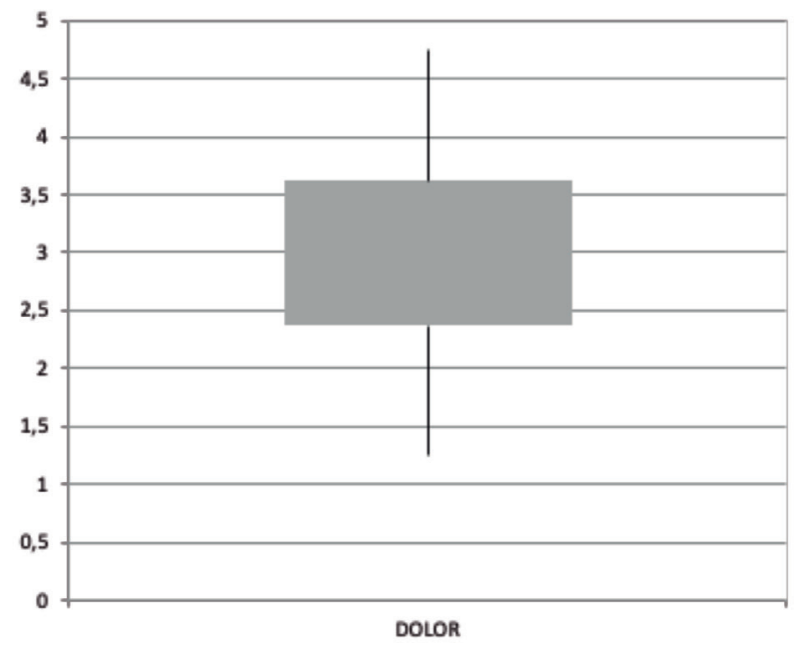

Gráfico Box Plot Intensidad de dolor para grupo control, según valores de la escala de EVA evaluados entre la primera y última sesión.

Fig. 4. Dolor grupo control según EVA.

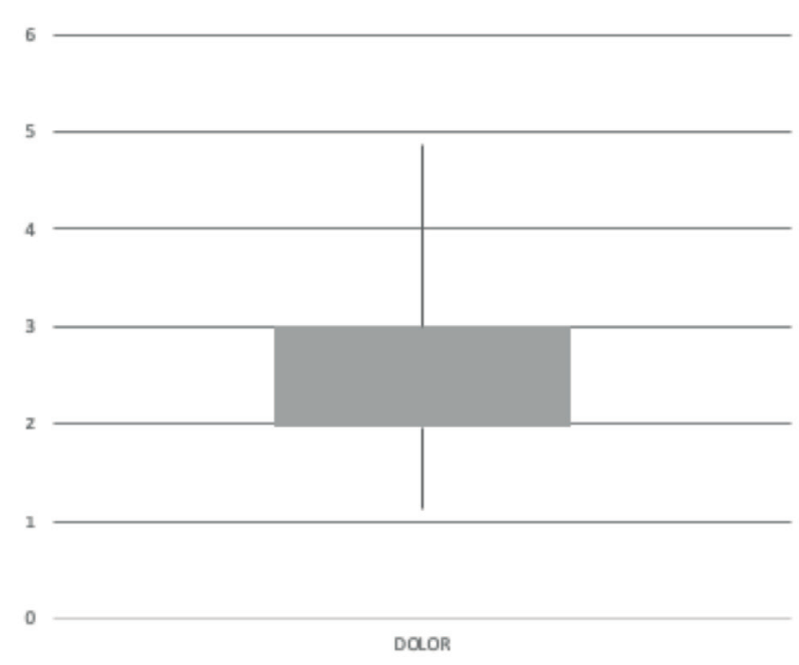

Gráfico Box Plot Intensidad de dolor para grupo experimental según valores de la escala de EVA evaluados entre la primera y última sesión.

Fig. 5. Dolor grupo experimental según EVA. 
po control, en contraste, mostró un aumento en las características miscelánea, afectiva y evaluativa, en su descripción del dolor, lo que podría hacernos pensar en que también se lograron cambios sobre su nivel de catastrofismo.

Los pacientes con dolor crónico presentan niveles elevados de catastrofismo $(13,14)$. El término catastrofismo hace referencia a una percepción mental negativa y exagerada respecto a la experiencia de dolor. Muchos de estos pacientes presentan creencias previas respecto al dolor, gran parte de la literatura científica sugiere que las principales consecuencias asociadas al catastrofismo son dolor más intenso, mayor consumo de analgésicos, disminución de las actividades diarias e incapacidad laboral $(13,14)$. Nosotros no hicimos distinción en el origen del dolor que presentaban los pacientes, solo excluimos a los que evidenciaran una lesión nerviosa asociada, por lo que la técnica del cruce de manos más los ojos cerrados podrían haber influido en estos resultados al generar cambios en su percepción de daño o amenaza. Legrain y Van Damme $(15,16)$ hablan sobre la influencia de la atención visual respecto a la percepción del dolor y dicen que si se centra la atención en los estímulos dolorosos, se podría exagerar la percepción del dolor, y que alejar la atención del estímulo doloroso, ya sea en otra tarea o quitando el campo visual, reduciría el dolor percibido por los pacientes, es por eso que creemos que los sujetos del grupo control que presentaron un aumento en las características de su dolor, probablemente haya sido a que mantuvieron una mayor visualización del estímulo que se les estaba aplicando.

Respecto al uso del TENS, nuevas investigaciones señalan que el dolor crónico no responde a un modelo convencional, sino más bien a un tratamiento más enfocado en la reorganización cortical. Es por esto que en este estudio ambos grupos, el experimental y control, tuvieron el mismo protocolo de tratamiento electroanalgésico, para no otorgar más resultados a la electroanalgesia nerviosa transcutánea, sin embargo, consideramos que el haber agregado un grupo al estudio que solo hubiera tenido el cruce de manos sin aplicación de TENS podría haber aportado mayor información, ya que el TENS, en este caso, pudo haber incidido en la disminución del dolor en ambos grupos y haber desestimado los resultados del grupo con cruce de manos.

Las fortalezas de la técnica de cruce de manos es que es una técnica que puede complementar otra terapia de modulación del dolor que se desarrolle en una extremidad unilateral, no tiene costo asociado, ya que solo se necesita cruzar las manos sobre la línea media del cuerpo y cerrar los ojos, y no es invasiva ni de riesgo para el paciente. Las debilidades que se deberían considerar para estudios futuros es agregar otro grupo de comparación en donde se aplique la técnica del cruce de manos, con ojos abiertos y sin el TENS, y también con otras terapias analgésicas periféricas tales como compresas húmedo calientes, masajes u otras, para evidenciar si las diferencias en la disminución de la intensidad del dolor es por la técnica del cruce de manos, que permita corroborar las alteraciones sensoperceptivas y cambios en el procesamiento de la información que les llega de la periferia a los pacientes con dolor crónico.

\section{CONCLUSIONES}

Los resultados no evidencian convincentemente que el cruce de manos potencia los efectos de la terapia analgésica en relación con la intensidad dolorosa. Más estudios serían necesarios para incluir esta técnica como una estrategia de apoyo en pacientes con dolor crónico, y poder manejar así las alteraciones sensoperceptivas y los cambios en el procesamiento de la información que les llega de la periferia.

\section{CONFLICTO DE INTERESES}

Los autores declaran no tener conflicto de intereses.

\section{AGRADECIMIENTOS}

A la Universidad por permitir la realización del estudio.

\section{BIBLIOGRAFÍA}

1. IASP, 1994. Classification of Chronic Pain, Second Edition, IASP Task Force on Taxonomy, edited by $\mathrm{H}$. Merskey and N. Bogduk. Seattle: ISAP Press; 1994. http://www.iasp-pain. org. Part III: 209-214.

2. Williams AC, Eccleston C, Morley S. Psychological therapies for the management of chronic pain (excluding headache) in adults. Cochrane Database Syst Rev. 2009. Cochrane Database Syst Rev 2012;11:CD007407. DOI: 10.1002/14651858.CD007407.pub3.

3. Sambo CF, Torta DM, Gallace A, Liang M, Moseley GL, lannetti GD. The temporal order judgement of tactile and nociceptive stimuli is impaired by crossing the hands over the body midline. Pain 2013;154(2):242-7. DOI: 10.1016/j. pain.2012.10.010.

4. Gallace A, Torta DM, Moseley GL, lannetti GD. The analgesic effect of crossing the arms. Pain 2011;152(6):1418-23. DOI: 10.1016/j.pain.2011.02.029.

5. Moseley GL, Flor H. Targeting cortical representations in the treatment of chronic pain: a review. Neurorehabilitation Neural Repair 2012;26(6):646-52. DOI: 10.1177/1545968311433209.

6. Di Pietro F, McAuley JH, Parkitny L, Lotze M, Wand $\mathrm{BM}$, Moseley GL, et al. Primary somatosensory cortex function in complex regional pain syndrome: a systematic review and meta-analysis. J Pain 2013;14(10):1001-18. DOI: 10.1016/j.jpain.2013.04.001.

7. Di Pietro F1, McAuley JH, Parkitny L, Lotze M, Wand BM, Moseley $\mathrm{GL}$, et al. Primary motor cortex function in complex regional pain syndrome: a systematic review and meta-analysis. J Pain 2013;14(11):1270-88. DOl: 10.1016/j.jpain.2013.07.004.

8. Moseley L. Unraveling the barriers to reconceptualization of the problem in chronic pain: the actual and perceived ability of patients and health professionals to understand the neurophysiology. J Pain 2003;4(4):184-9.

9. Swart CM, Stins JF, Beek PJ. Cortical changes in complex regional pain syndrome (CRPS). Eur J Pain 2009;13(9):902e7. DOI: 10.1016/j.ejpain.2008.11.010.

10. Serrano-Atero MS, Caballero J, Cañas A, García-Saura PL, Serrano-Álvarez C, Prieto J. Valoración del dolor (II). Rev Soc Esp Dolor 2002;9(2):109-21. 
11. Melzack R. The McGIII Pain Questionnaire: major properties and scoring methods. Pain 1975;1(3):277-99

12. Aglioti S, Smania N, Peru A. Frames of reference for mapping tactile stimuli in brain-damaged patients. J Cogn Neurosci 1999;11(1):67-79.

13. Sullivan MJL, Bishop SR, Pivick J. The pain catastrophizing scale: Development and Validation. Psychological Assessment 1995;7(4):524-32. DOI: 10.1037/1040-3590.7.4.524.

14. Sullivan MJL, Thorn BE, Haythornthwaite JA, Keefe F, Martin M, Bradley L, et al. Theoretical perspectives on the relation between catastrophizing and pain. The Clin J Pain 2001;17(1):52-64.

15. Legrain V, Guerit JM, Bruyer R, Plaghki L. Electrophysiological correlates of attentional orientation in humans to strong intensity deviant nociceptive stimuli, inside and outside the focus of spatial attention. Neurosci Lett 2003;339(2):107-10.

16. Van Damme S, Legrain V, Vogt J, Crombez G. Keeping pain in mind: A motivational account of attention to pain. Neurosci Biobehav Rev 2010;34(2):204-13. DOl: 10.1016/j. neubiorev.2009.01.005. 